\title{
Application of Edge Detection for Vehicle Detection in Traffic Surveillance System
}

\author{
Ashwini. B \\ Associate Professor \\ Dept of ISE, NMAMIT, Nitte \\ (Affiliated to VTU, Belagavi) \\ Karkala, India
}

\author{
Yuvaraju B N, Ph.D. \\ Professor \\ Dept of CSE, NIE, Mysore \\ (Affiliated to VTU, Belagavi) \\ Mysore, India
}

\begin{abstract}
Detecting the vehicles and having a detailed behavior analysis of the vehicles and their behavior in a traffic surveillance system is an emerging area of research. Vehicle detection would be the first step to be addressed in this process. Various classes of vehicles are to be detected from the surveillance video and then they need to be classified based on various feature points. This paper brings out the different methods used for the vehicle detection from a video. An overview of the edge detection methodology is also given here, which is one of the methodologies used in vehicle detection.
\end{abstract}

\section{General Terms}

Image processing, Computer Vision.

\section{Keywords}

Intelligent Transport System, Background subtraction, optical flow, frame differencing, edge detection

\section{INTRODUCTION}

With the growth in the economy of a country, the income level of urban population has increased. Also due to industrialization, the number of vehicles on the roads of cities and highways are also increased in last few years. This has contributed to severe congestion problems on roads throughout the country. Most of the road infrastructures are often not equipped to deal with the heavy traffic flow, which in turn creates unsafe road conditions. One of the important ways of improving safety on road is to have a sound traffic management system: a modern system that can monitor, manage as well as enforce traffic rules efficiently.

Intelligent transport system (ITS) is an advanced application which aims to provide innovative services relating to different modes of transport and traffic management and enable various users to be better informed and make safer, more coordinated, and 'smarter' use of transport networks. Traffic-flow measurement and automatic incident detection using video camera is a form of vehicle detection.

Cameras are being installed on the roads and traffic signals for monitoring the vehicles and to monitor breaking of traffic rules. Since video based detection systems such as those used in automatic number plate recognition do not involve installing any components directly into the road surface or roadbed, this type of system is known as a "non-intrusive" method of traffic detection. Video captured from camera is fed into processors that analyze the change in the characteristics of the video frames as vehicles pass. The cameras are typically mounted on poles or structures above or adjacent to the roadway. A single video detection processor can detect traffic simultaneously from one to eight cameras, depending on the brand and model. The typical output from a video detection system is lane-by-lane vehicle speeds, counts, and lane occupancy readings.[1] Some systems provide additional outputs including gap, headway, stopped-vehicle detection and wrong-way vehicle alarms. Edge detection method is one of the approaches in the object detection. Vehicle detection is one of the applications of object detection. In this, the shape, size and location of the detected object plays a major role in decision making for the action to be taken for traffic surveillance system

\section{RELATED WORK}

Various algorithms have been proposed in the literature for vehicle detection in traffic videos. The techniques include background subtraction, frame differencing, optical flow and Edge Detection Method [1]

Background Subtraction- In this, initially a background model is created. The foreground objects are then detected by subtracting the background from current frame. However, the background is extremely sensitive to poor environments and easy to be blurred. In heavy traffic conditions, for example, if the road area is severely covered with frequently moving vehicles, then it is very difficult to model the background [2]. Even though many researchers have adopted this method for object detection, none of their researches have given an effective result of object detection using this method

Frame Differencing - The philosophy of frame differential method is to exploit the difference between two successive frames or a few frame images for target detection. General steps are following: firstly, calculate the difference between the $\mathrm{k}$ and $\mathrm{k}-1$ frame in the video image sequence to get the difference image; then, select a threshold and transform the difference image into binary value. When the pixel value in difference image exceeds the given threshold, the pixel is treated as sports pixel, conversely as background pixel. As for the sports image after binary transformation to the difference image, in order to eliminate the hollow pixel, connectivity is performed on the sports pixels. A size threshold is given, when a connected area is greater than this size, it indicates a moving target.

Frame Differential method is a simple algorithm and easy to implement. Nevertheless, this algorithm also has some disadvantages. Owing to the direct differential of the adjoining frames, the obtained difference image often contains a lot of noise, hollow and split parts. Therefore, in order to ensure the accuracy of the detection, often we need do perform some post-processing using some method more complex than frame difference algorithm for supplement. Commonly used algorithms include filling algorithm that eliminates the hollow and connects the parts. Size filter, is 
another approach [1] which filters out the connected parts, which are smaller than a certain size, as the connected components of moving vehicle would not be so small. In size filter, threshold is the determinant element of detection accuracy. In recent years, some researchers suggest adopting adaptive statistical optimal binary method to determine the size threshold, for the distinction of sports target and background image, which overcomes the shortcomings of poor generality using fixed threshold

Comparing with background modeling, frame differencing method is more robust and not sensitive to illumination change. This method extracts moving objects using two frame differencing method. Researchers have shown that with three frames differencing method it still performs well even with slight shake of the camera

Optical flow - Optical flow is a technique used to get the motion of objects in video. Optical flow calculates a velocity for points within the images, and provides an estimation of where points could be in the next image sequence. Typically the motion is represented as vectors originating or terminating at pixels in a digital image sequence. When relative motion occurs between the human eye or camera and observed target, the velocity vectors in $3 \mathrm{D}$ scenery, after projection in imaging plane, will form the two-dimensional velocity vector - optical flow field.

Optical flow field not only includes the movement information of target observed, but also carries the three dimensional structure information of the related scenery, which can be used to determine the movement condition relative to the observer target and the observed objects.

The optical flow evaluation based motion detection takes advantage of optical flow characteristic that the moving object changes with the time. No preprocessing is required in the optical flow method, which makes possible for the computation to be preformed directly on the original image. Optical Flow Method's advantage also lies in the camera even under the moving condition can detect the moving targets.

Though there are various algorithms to detect the object, each one has its advantages and disadvantages. Following are the observations made regarding the above mentioned methods.

In background subtraction method, background has to be updated at regular intervals. Handling of complex backgrounds and lighting changes is a challenge. Also the background is influenced by shadows. The performance of this method diminishes when the scene involves dynamic elements or occluded fronts, such as waving trees, flocks of birds, rippling water, fog or smoke.[3]

The challenges faced by frame differencing method are- the detected region is union of object regions in adjacent frames, which makes it difficult to identify the required object. This method will fail in the situations with slowly moving objects. If objects have little texture, there will be many holes in the detected region.[4]

In optical flow method, most of the optical flow calculation methods are very complex and their anti-noise performance is poor. This method is time-consuming and real-time problem should be treated specially. So it is usually combined with other detection methods [4].

\section{EDGE DETECTION METHODOLOGIES}

An edge is defined as a sharp change in the pixel values in an image. Edge detection is another widely used methodology in image processing and computer vision applications for detecting the discontinuity. These discontinuities are due to abrupt changes in pixel intensity which characterizes boundaries of objects in a scene. Edges provide boundaries between different regions in the image. These boundaries are used to identify objects for segmentation and matching purpose. These object boundaries are the first step in many of computer vision algorithms like edge based face recognition, edge based obstacle detection, edge based target recognition, image compression etc. There are many edge detection operators available, which is used to extract edges from the image [1]. The edge detection operators will identify vertical, horizontal, corner and step edges. The quality of edges detected by these operators is highly dependent on noise, lighting conditions, objects of same intensities and the density of edges in the scene. Depending on the edges considered, the parameters in the edge detector can be adjusted along with the change in the threshold values, for better performance.

There are many methods to make edge detection. The most common method for edge detection is to calculate the differentiation of an image. The first-order derivatives in an image are computed using the gradient, and the second-order derivatives are obtained using the Laplacian.

Classical edge detection [1] method is to construct the edge detection operator, to a small neighborhood of pixels in the original image,

Where $\nabla f(x, y)=\frac{\partial f}{\partial x} i+\frac{\partial f}{\partial y} j$ the gradient of the image is, $\nabla f(x, y)$ contains grayscale information. $e(x, y)=\sqrt{\left(f^{2}\right.} x+$ $\left.f^{2} y\right)$ is the gradient of $\nabla_{f}(x, y), e(x, y)$ can be used as edge detection operator. In order to simplify the calculation, $e(x, y)$ can also be defined as the sum of the absolute value of the partial derivative $f_{x}$ and $f_{y}$ :

$$
e(x, y)=\left|f_{x}(x, y)\right|+\left|f_{y}(x, y)\right|
$$

A number of algorithms are proposed based on these theories. The edge detection methods that are commonly used are: Roberts edge detection operator, Sobel edge detection operator, Prewitt edge detection operator, canny edge detection operator, Laplace edge detection operator. These operators are very different in the algorithm complexity and performance. A suitable operator has to be selected based on the actual situation. The edge detection algorithm in time measure is obtained by calculating the difference between the corresponding pixels for continuous frame. However, the edge detection operators above are essentially a high-pass filter, which helps in sharpening the image. This enhances the edge while also expanding the high-frequency noise of edge degradation.

The gradient based detectors [7] viz. Sobel, Robert, Prewitt, convolve the input image with their respective convolution mask to generate a gradient image. The output of these edge detectors is very much sensitive to the threshold.

Robert operator uses following $2 \times 2$ two kernels:

$$
D_{x}=\left[\begin{array}{cc}
1 & 0 \\
0 & -1
\end{array}\right] \text { and } D_{\mathrm{y}}=\left[\begin{array}{cc}
0 & 1 \\
-1 & 0
\end{array}\right]
$$


The positive factor of this operator is its simplicity but having small kernel it is highly sensitive to noise and not much compatible with today's technology.

Sobel uses following $3 \times 3$ two kernels:

$$
\begin{aligned}
D_{i}=\left[\begin{array}{lll}
-1 & 0 & +1 \\
-2 & 0 & +2 \\
-1 & 0 & +1
\end{array}\right] \text { and } \\
D_{j}=\left[\begin{array}{ccc}
1 & 2 & +1 \\
0 & 0 & 0 \\
-1 & -2 & -1
\end{array}\right]
\end{aligned}
$$

Canny edge detector have advanced algorithm derived from the work of Marr and Hildreth. It is an optimal edge detection technique as it provides good detection, clear response and good localization. It is widely used in current image processing techniques with further improvements.

Foreground detection method [5] based on edge detection is usually more efficient. When an image brightness changes, its outlook is stable, because edge information is still obvious even in dim lighting environments. Hence this method is applicable for vehicle detection at night.

R. K. Satzoda et.al [6] proposed an efficient technique to detect vehicle queue lengths at traffic junctions based on progressive block based image processing. They also proposed a two-step approach for vehicle detection that relies on edges and dark features in the image. The process is divided into two steps. The first step of processing uses edge detection with a static threshold; the second step detects dark vehicle features as an additional indication of vehicle presence. The second step of processing uses averaging as a dynamic threshold method and hence eliminates the need for complex threshold adjustments in edge detection. The Sobel edge detector is used in the first step of processing to generate the edge maps of each Block of Interest in every lane. The Sobel edge detector detects discontinuities in image intensities, which indicate edges in an image. The number of edge pixels in the edge map of Block of Interest is compared with an experimentally computed threshold. A high amount of edge content in the edge map of the Block of Interest is an indication of vehicle presence in that Block of Interest because the road surface of an unoccupied. The experiment results in the paper have proved that the two-step approach for vehicle detection is robust to heavy and light shadows. Further, the threshold adapts itself dynamically to handle varying light conditions.

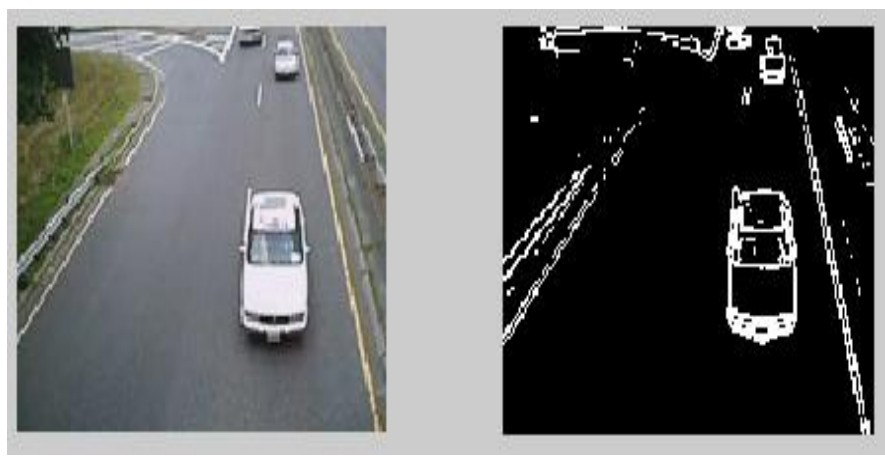

(a)
Wen-Kai Tsai et al [8] proposed an algorithm to extract the horizontal edge as a composition of vehicles by using Sobel filters and orientation gradient calculations. Firstly, to reduce the memory consumption and computation time, regions of interest (ROI) are defined based on the scene and camera type. A Sobel filter and thresholding are applied to extract the horizontal edge from a gray-level image, which then subsequently yields the binary horizontal edge-detected image. Lastly, the Sobel mask and its gradients are applied to calculate the orientation at every pixel in the ROI.

Jingwei Liu et.al [9] proposed a new edge extraction approach for vehicle capturing and counting. In this approach, a neighbourhood gradient prediction (NGP) is employed. NGP predictor uses neighbourhood comparison information to produce the current pixel edge values. The result is compared based on neighbourhood comparison information, if neighbourhood comparison valued higher than the threshold computed by the numbers of the neighbourhood, a weight valued will be given which depend on its direction. NGP edge detector is proved to be simple and effective approach to extract edges in an image and it is insensitive to noise in the image because of its multi-direction. The edges detected using this approach were thick, which would not be suitable for some applications where the detection of the outmost contour of an object is required

With the advantages of edge detection, there are few challenges also. A wide range of operators are available that can extract the edges from noisy image. But these edges are less accurate. That is due to the presence of noise they extract false edges. They do not find the boundaries of object having small change in intensities values. That result in poor localization of edges. So, a suitable operation is to be performed to identify such a gradual change in intensities. So there are problems of false edge detection, problem due to noise, missing of low contrast boundaries, high computational time etc.

\section{EXPERIMENTAL RESULTS}

Experiments were conducted to detect vehicles in a video. A traffic video of $8 \mathrm{sec}$ was initially divided into frames. Edge detection algorithm is applied on the frames as shown in Fig 1. Sobel edge detection operator is used in the edge detection algorithm.

Fig 1: Original image and edges of the same 


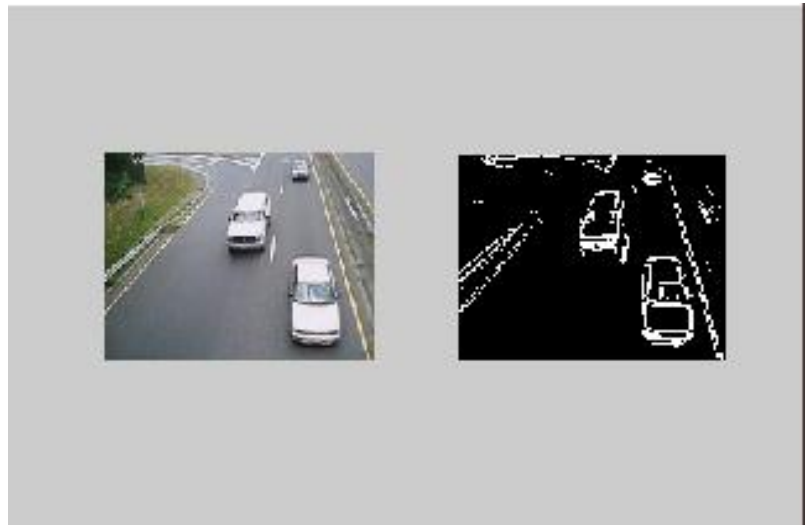

(a)

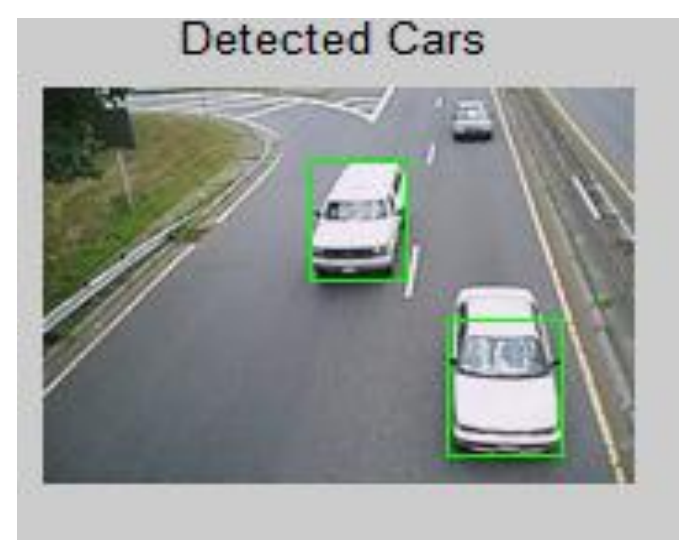

(b)

Fig 3: Edge detection of two cars

Once the edges are detected, the objects or the connected components are detected using the blob analysis, which computes statistics for connected regions in a binary image. This results in detecting false objects also. The detected false objects are deleted by computing their area. Considering a threshold value of the area, false objects are removed by eliminating the objects less than the threshold. Fig 2 shows the final detected object. Fig 3 shows the original frame and the edge detected objects. Fig 4 shows two cars detected in the frame. This illustrates the detection of more than one vehicle in the same frame

\section{CONCLUSION}

Edge detection is one of the initial steps to detect vehicles. The paper also discusses on other methods of vehicle detection. Once Edges are detected the image is fed to the next module for finding the connected components. Using the parameters of the detected connected components, we remove the false detected objects. Thus the vehicles are detected in a video using edge detection methodology. This detected vehicle information may be further used for analyzing the behavior of the vehicle.

\section{REFERENCES}

[1] Kun Wu, Haiying Zhang, Tianmao $\mathrm{Xu}$ and Ju Song, 2011 Overview of Video-Based Vehicle Detection Technologies,6th International Conference on Computer Science \& Education (ICCSE 2011)

[2] Qiong Cao, Rujie Liu, Fei Li and Yuehong Wang. 2010 An Automatic Vehicle Detection Method Method Based on Traffic Videos. In Proceedings of IEEE 17th International Conference on Image Processing.
[3] B. Karasulu and S. Korukoglu, "Performance Evaluation Software", SpringerBriefs 7 in Computer Science, DOI: 10.1007/978-1-4614-6534-8_2, () The Author(s) 2013

[4] Bin Tian, Qingming Yao, Yuan Gu, Kunfeng Wang and Ye Li,2011 Video Processing Techniques for Traffic Flow Monitoring: A Survey, 14th International IEEE Conference on Intelligent Transportation Systems Washington, DC, USA.

[5] Zhang Yuanyuan, Zhang kaiwen and Mao Yuming, 2012 Research on Vehicle detection method based on video Images, in International Conference on Industrial Control and Electronics Engineering, IEEE

[6] R. K. Satzoda, S. Suchitra, T. Srikanthan and J. Y. Chia, 2011,Vision-based Vehicle Queue Detection at Traffic Junctions, IEEE Conference on Industrial Electronics and Applications (ICIEA).

[7] Saket Bhardwaja and Ajay Mittalb,2012, A Survey on Various Edge Detector Techniques, in Procedia Technology 4, Published by Elsevier Ltd, pp 220 - 226,

[8] Wen-Kai Tsai, Shao-Lung Wu, Li-Juo Lin, Tse-Min Chen and Ming-Hua Li, 2014 Edge-based Forward Vehicle Detection Method for Complex Scene, ICCE .

[9] Jingwei Liu, Yong Zhao, Yule Yuan, Wei Luo and Kai Liu,2011,Vehicle Capturing and Counting Using A New Edge Extraction Approach, International IEEE Conference on Intelligent Transportation Systems Washington, DC, USA. 